\title{
Throughput analysis in mobile adhoc network: a literature survey
}

\author{
Madhurya Kodialbail Divakara ${ }^{1}$ and Dr. A. R. Abdul Rajak ${ }^{2}$ \\ ${ }^{1}$ Department of Electronics and Communications Engineering, Bits-Pilani Dubai Campus, UAE \\ ${ }^{2} \mathrm{HOD}$ of Electronics and Communications Engineering, Bits-Pilani Dubai Campus, UAE
}

\begin{abstract}
A rapid advancement in the area of mobile networks has been observed over the recent years due to the spread of reasonable, existing wireless devices. Hence it has given way to numerous opportunities to many researchers to work on Mobile Adhoc Networks. There are several key challenges in implementing the MANETS. It does not use any existing infrastructure or centralized administration. Each mobile node acts as a router. Due to its dynamic features, they are endangered. This paper provides a detailed study and throws light on the MANETS Topology in Wireless Adhoc Networks and focusses on performance parameters throughput, end to end delay and network load, its effect on the performance of the MANET network depending upon the number of users.
\end{abstract}

\section{Introduction}

Wireless Adhoc Networks are peer to peer wireless networks where wireless devices are able to communicate directly with each other. They have a Base Service Set (BSS) without an Access Point (AP) in order for communication to take place. They neither rely on any pre-existing infrastructure nor use any set of central administrations to follow.

There are 2 main types of Wireless Adhoc Networks i.e. Infrastructured and Infrastructureless. In Infrastructured Networks, a node can move while communicating and the base stations are fixed. As the node elopes out of range from the base station, it merges into the range of another station, whereas in Infrastructureless Networks, the base stations are not fixed. From the above mentioned they can be classified as MANETS (Mobile Adhoc Networks), WMNS (Wireless Mesh Networks), WSNS (Wireless Sensor Networks) and HWNS (Hybrid Wireless Networks).

MANET is a self-configuring infrastructure with fewer networks of mobile devices connected by wireless connections. Such networks may also be connected to the larger Internet. They are dynamically formed by an absolute system of mobile nodes and are made to move freely, randomly hence leading to the change of the network topology occasionally. Besides being in a routable networking setting, MANETS are on the top of a Link Layer model.

In MANETS, nodes within a wireless transmission range are able to communicate precisely ; however, nodes out of its range have to rely on alternative nodes to relay messages. Thus, a multi-hop scenario occurs, where several transitional hosts relay the packets sent by the source host to take it to its destination node. MANETS are capable of multi-hop routing. It is also able to allocate and 
maneuver security, routing and host configuration. A malicious behavior highly resembles another benign behavior.

Mobile nodes are evolved with lesser memory, power and light weight characteristics. Due to their impulsive behavior it burdens human involvement to configure the network. The nodes have exact features with related responsibilities and competences and thereby enable an entirely symmetric environment. They have immense user density and user mobility.

Types of MANETS includes : VANETS (Vehicular Adhoc Networks), In-VANETS (Intelligent Vehicular Adhoc Networks) and i-MANETS (Internet Based Mobile Adhoc Networks).

VANETS (Vehicular Adhoc Networks) facilitates an effective communication with another vehicle and is free to communicate with roadside equipment.

InVANETS (Intelligent Vehicular Adhoc Networks) uses artificial intelligence to challenge unexpected situations like vehicle collisions and accidents.

i-MANETS (Internet Based Mobile Adhoc Networks) provides a link for both fixed and mobile nodes.

Some challenges in MANETS encountered are that these wireless link features are timefluctuating in nature; there are transmission impairments like fading, path loss, blockage and noise that increases the vulnerability of wireless channels, limited range of wireless transmissions causes the narrow radio band to reduce the data rates. Hence optimized usage of bandwidth is mandatory by keeping low overhead. Other challenges also include packet losses due to errors in transmission, route changes due to mobility and frequent network partitions.

There are 2 types of routing protocols in MANET. Proactive routing protocol is where nodes endlessly calculate and update routes. Periodic \& triggered updates occur when a link modifies. This method is productive if the routes are often used, in order to keep up-to-date information about the network routes. It is quite similar to conventional routing protocols. Each node sustains steady and upto-date routing information in the form of a table with the next-hop to reach all nodes in the network. These include DSDV (Destination Sequenced Distance Vector), CGSR (Cluster Head Gateway Switched Routing). Reactive routing protocol is where nodes evaluate and alter routes whenever needed. When a node has a packet to send, it checks to see if it has a valid route. If no valid route is known, then node must send out a route-request message to obtain a valid route. This method is efficient if routes are not used often. These include AODV (Adhoc On Demand Distance Vector), DSR (Dynamic Source Routing).

QOS is a depiction and measurement of the overall performance of a service, such as computer network, a cloud computing service and the performance seen by the network users. QOS also provides support to Mobile Adhoc Networks, by supporting internet directly. There is a difficulty in getting and managing the link states directly because they change with surrounding circumstances and also have certain bandwidth constraints.

The main QOS parameters in the network are :

Throughput- The rate of successful message delivery over a transmission.

Latency- The amount of time taken for the message to traverse a system.

Packet Loss- The number of packets lost during the transmission process.

Jitter- The variation in the delay of received packets.

Reliability - Percentage of packets discarded by the user.

Bit Rate- The number of bits per second that can be transmitted along a digital network.

End-to-end delay- The time taken for a packet to be transmitted across a network from source to destination.

\section{Related Works}

This section presents some previous works related to throughput and end to end delay performance analysis on MANETS. Adhoc Networking has become one of the most interesting research fields inside the networking. 
H. Hallani, et al. [1] in 2005 proposed in their paper about creating a small physical network, gathered experimental results and compared those with simulations through OPNET. The simulation results showed good agreements with those obtained through the experiments conducted physically. It also showed that a linear drop in the throughput, with reference to the number of nodes simultaneously connected to the same destination.

There are also some interesting references based on real applications, so Monika Rajput [2], et al. have highlighted in their research paper about the various reactive routing protocols such as Ad hoc On-demand Distance Vector (AODV), Dynamic Source Routing (DSR) and Temporally Ordered Routing Algorithm (TORA) and compared them on the basis of throughput by increasing number of nodes in the network.

There is an interesting approach that researchers are currently investigating in the application of reinforced learning in routing protocols [3]. For e.g. Mostafa Fazeli et al. presented in their research paper about throughput analysis using 10,15 and 20 users without using any routing protocols through OPNET software. These researchers extensively used graphs to highlight their point of view. From the simulations it was observed that as the number of users keeps increasing then the throughput of the network increases.

Ikramullah Khosa et al. [4] evaluated in their paper by providing a detailed analysis of IEEE 802.11 medium access control (MAC) protocol. The performance of CSMA/CA protocol is studied in depth by using optimized network engineering Tool (OPNET) with different sets of WLAN parameters. Simulation results have been obtained in different network scenarios by changing the number of stations in the network. This was also analyzed for adhoc and infrastructure networks.

Fan Ya-qin et al. [5] suggested a better solution to overcome problems of inefficiency in large expansion of adhoc routing protocols through DSR routing protocol and concluded that DSR protocols are suitable for only small scale MANET networks. Simulation results thus have obtained references for further research \& study.

Thodeti Srikanth, et al. [6] besides doing a bit of introduction in their research paper mainly considered different methods like routing load, end to end packet delivery and performance of protocols and used http and low load traffic. These researchers concluded that the efficiency of a network can be achieved by selecting efficient protocols based on the network requirement. This research article mainly explained about the simulator OPNET Modeler, its architecture and performance parameters to a certain extent.

In their paper N.S. Samshette, et al. [7] calculated about throughput, end to end delay, packet delivery ratio in order to evaluate the performance of different mobility models; Random Walk, Random Waypoint, Random direction, Gauss Markov, Manhattan Grid through routing protocols. By using Random Walk AODV model high throughput and packet delivery ratio were produced leading to low end to end delay as compared to Random Waypoint and Reference Point Group Mobility model. Hence this also led to the explanation of relevant points on hybrid on demand protocols by the researchers.

Beata Krupanek et al. [8] presented a detailed study on the quality of service for modern wireless networks and had also explained relevant details about multimode network simulations in the OPNET and discussed about the prime ways in ensuring proper transmission of quality measurement in wireless sensor networks.

P.Venkata Maheswara et al. [9] in their paper compared about the various routing protocols for MANETS i.e. AODV, DSR, TORA and OLSR. Its performance was evaluated by considering the parameters such as routing overhead, delay, throughput and network load by varying the mobile nodes.

Parulpreeth Singh et al. [10] highlighted the performance of the DSR reactive routing protocol under traffic http, video conferencing, ftp and email. On the basis of evaluation the performance parameters such as throughput and end to end delay were taken into consideration and they concluded which traffic delay would be best for those parameters.

Sukhwinder Kaur et al. [11] in their paper had shown a comparison regarding performance parameters in terms of delay, throughput and media access delay by using the routing protocols AODV, OLSR and DSR also studied the network performance. The application traffic taken into 
consideration were http and data base. They wanted to check which protocol gave the best result for their study.

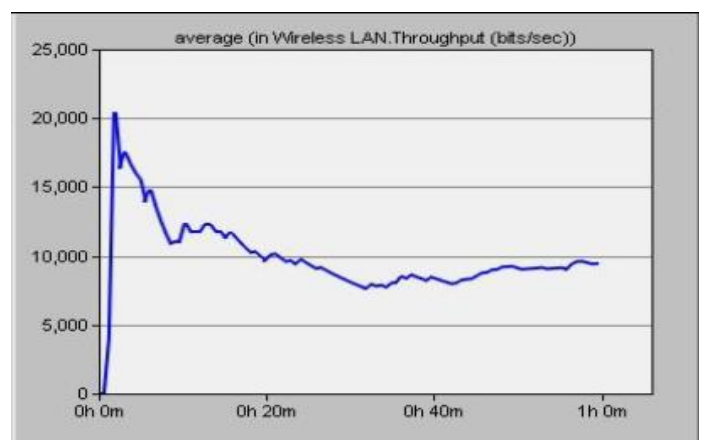

Figure. 1 Throughput Graph for 10 users. (Fig 1, [3])

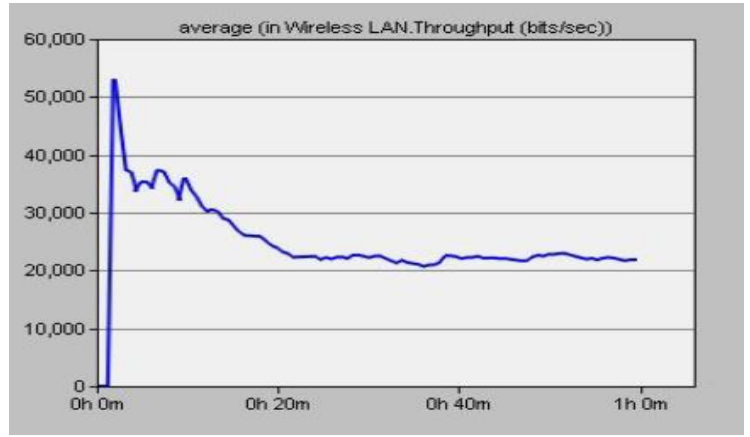

Figure. 2 Throughput Graph for 15 users. (Fig 2, [3])

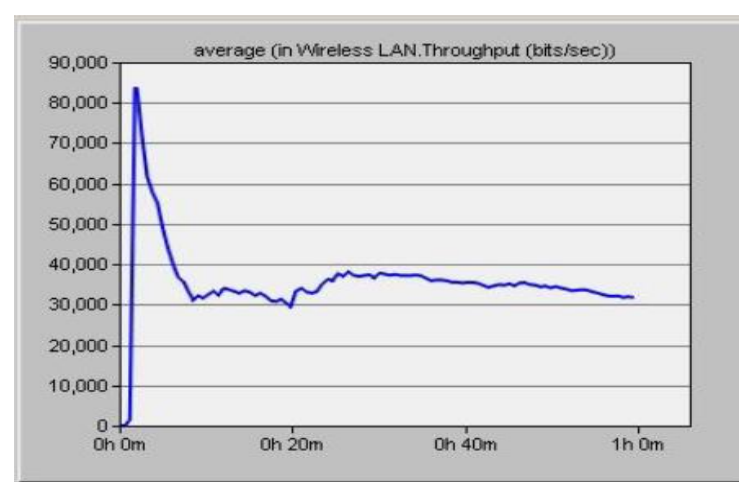

Figure. 3 Throughput Graph for 20 users. (Fig 3, [3]) 


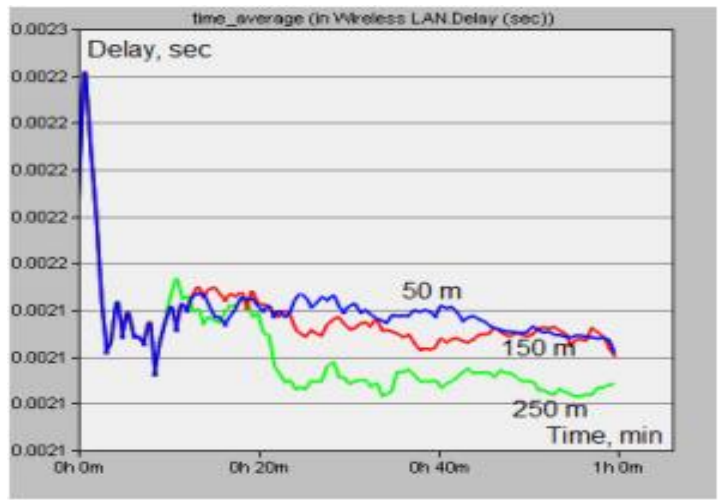

Figure. 4 Delay vs. node distance for DCF (Distributed Coordinated Function) algorithm. (Fig 4, [8])

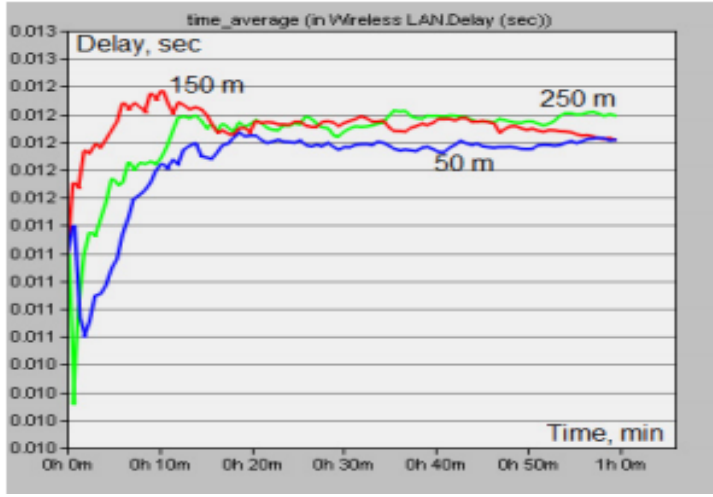

Figure. 5 Delay vs. node distance for PCF (Point Coordinated function) algorithm. (Fig 5, [8])

From the above figures it can be observed that the throughput keeps increasing as the number of users keep increasing as illustrated in Figure. 1, Figure. 2 and Figure. 3 but in Figure. 4 and Figure. 5 it can be seen that the node distance and delay are indirectly proportional in DCF algorithm, whereas in PCF algorithm distance and delay are proportional to each other. Hence we say that PCF mechanism affects delay much more than DCF.

\section{Results}

H. Hallani, et al [1] concluded by evaluating the performance of adhoc networks through simulation and analysis. Simulation results confirmed the results that matched with the ones obtained through physical experiments. It has also showed that when the signal strength between source and destination is weak, routing the traffic will take place through an interconnecting node and leads to greater values of throughput.

Monika Rajput [2], et al concluded by comparing all the protocols and through the results commented that TORA performs better than AODV and DSR as the number of nodes keeps increasing. The performance of the network was evaluated for throughput explicitly.

Mostafa Fazeli et al. [3] compared the throughput parameters for different scenarios when the number of users were 5,10 and 15. Through the simulation graphs they observed and analyzed that as the number of users increase then throughput also increases.

Ikramullah Khosa et al. [4] concluded by analyzing the performance of channel access protocol under varied conditions of the network. By simulating through OPNET they provided good views that performance was better if packet size was small and it was better performing under Adhoc mode. The performance of the protocol can be upgraded by using adaptive algorithm. 
Fan Ya-qin et al. [5] concluded by conducting simulations of DSR protocol through a broad range of distinctive networks. It was better to be used when the topology of the network was small and showed complexities when the topology of the network changed and when the network size increases continuously. DSR will show a number of networks performance problems. Some kinds of improvements are needed to be made to upgrade the DSR protocol to easily adapt to the changes in network topology.

Thodeti Srikanth, et al [6] completed their research by giving and ensuring an insight about the simulation based approach on MANET and Adhoc. They also explained about the software in great depth.

N.S. Samshette, et al [7] concluded by using different mobility models for analyzing different parameters such as throughput, end to end delay, packet ratio with different routing protocols as AODV, TORA, DSR and AOMDV. Through these results it was finally proved that Gauss Markov Model has better results as compared to Manhattan Grid Model.

Beata Krupanek et al. [8] summarized and concluded that if DCF (Distributed Coordination Function) was used then it would cause high latency depending on the type of traffic. If PCF (Point Coordinated Function) was used it would work for all nodes and provides a good overall performance. P.Venkata Maheswara et al. [9] evaluated and concluded by explaining the protocols AODV, DSR, TORA and OLSR ad-hoc routing protocols under different load and different number of users. The software used is OPNET Modeler 14.0 and simulations with varying traffic were run for $3600 \mathrm{sec}$.

Parulpreeth Singh et al. [10] concluded their research by considering various load conditions and checked which one performed the best for throughput and end to end delay. So it was found that http showed best performance in terms of both throughput and delay. In video conferencing throughput was the highest and was lowest in http.

Sukhwinder Kaur et al. [11] evaluated and concluded that in data base traffic, OLSR achieved highest throughput, least delay and least media access delay. In http traffic, OLSR performed well. So, it was finally observed from their research that OLSR performed the best compared to other two protocols.

\section{Conclusions}

From the above 11 surveys and researches carried out by various researchers it summarizes, an overview of Mobile Adhoc Networks (MANETS), its applications and characteristics that distinguishes it from other wireless networks. Due to its characteristics, there is a need of separate routing protocols for MANET. Classification of routing protocols for MANET has been done on the basis topology of the network i.e. proactive or table-driven and reactive or demand-driven. A summarized overview of routing protocols belonging to each type of classification has also been presented above hoping that it will be useful and helpful to students and researchers in this field. From this, it is concluded that MANET routing protocols are designed based on the application area and environment and it is not possible to design a single protocol, which is suitable for all MANETS in all situations.

\section{References}

1. Hallani, S.A. Shahrestani and F.H. Stootman, "Wireless Adhoc Networking: Analysis and Simulation of Validation Results," TENCON 2005 IEEE Region 10.

2. M. Rajput, P. Khatri, A. Shastri and K. Solanki, "Comparison of Ad-hoc Reactive Routing Protocols using OPNET Modeler", International Conference on Computer Information Systems and Industrial Management Applications (CISIM), 2010 IEEE, 530-534.

3. M.F.H. Vaziri, “Assessment of Throughput Performance under OPNET Modeler Simulation Tools In Mobile Adhoc Networks (MANETS)”, 2011 Third International Conference on Computational Intelligence, Communication Systems and Networks, 328-331. 
4. I. Khosa, U. Haider and H. Mosood, "Evaluating the Performance of IEEE 802.11 MAC Protocol using OpNET Modeler", 2010 International Conference on Electronics and Information Engineering, 2, 91-95.

5. F. Ya-qin, F. Wen-yong and W. Lin-Zhu, "OPNET- Based Network Of MANET Routing Protocols DSR Computer Simulation”, 2010 WASE International Conference and Information Engineering,46-49.

6. T. Srikanth and Dr.V.B. Narsimha, "Simulation- Based Approach to Performance Study of Routing Protocols in MANET and Adhoc Networks", IJCSNS, 11,111, (September 2011).

7. N. S. Samshette, A.D.Bhoi and R.D.Kharadkar, "Analysis \& Comparison of Mobility Models for Ad-hoc Network", IJRITCC, 2, 1357 - 1362, (May 2014).

8. B. K. R. Bogacz, “ OPNET Modeler Simulations of Performance for Multinodes Wireless Systems",IJMQE, 2-5.

9. P.V. Maheswara and K. B. Naik, "Routing Protocol Performance Issues and Evaluation Considerations in MANET", IJERA, 3, 1-5, (Jul-Aug 2013).

10. P. Singh, E. Barkhodia, G. K. Walia, "Evaluation Of Various Traffic Loads in MANET with DSR Routing Protocols Through OPNET", IJDPS, 3,75-83, (2012)

11. S. Kaur, R.K. Bansal and S. Bansal, "Performance Analysis Of AODV, OLSR and DSR Routing Techniques For Adhoc Mobile Networks”, IJCSEIRT, 3, 195-200, (Dec 2013). 